\title{
Temperature and Aging Dependence of Strain-induced Crystallization and Cavitation in Highly Crosslinked and Filled Natural Rubber
}

\author{
Quentin Demassieux ${ }^{1}$, Daniel Berghezan ${ }^{2}$, Sabine Cantournet ${ }^{3}$, Henry Proudhon ${ }^{3}$ and Costantino \\ Creton $^{1 *}$ \\ ${ }^{1}$ Laboratoire de Sciences et Ingénierie de la Matière Molle, ESPCI Paris, PSL University, Sorbonne \\ University, CNRS, F-75005 Paris, France. \\ ${ }^{2}$ Michelin Research Center, Ladoux, Clermont-Ferrand, France \\ ${ }^{3}$ Centre des Matériaux, Ecole des Mines, Evry, France
}

Keywords : Natural rubber, X-ray scattering, Mechanical Properties, Crystallization, Cavitation

\begin{abstract}
We have investigated the structural changes occurring in highly crosslinked and carbon-black filled natural rubber under uniaxial extension by small and wide angle X-ray scattering using synchrotron radiation. The experiments focused on strain-induced crystallization (SIC) and nanocavitation and were carried out on a model series of materials as a function of temperature and aging conditions. We find that for all materials both SIC and cavitation decrease markedly with temperature and ageing. However the presence of carbon black filler shifts the ceiling temperature where SIC is observed to at least $120^{\circ} \mathrm{C}$, presumably by a nucleating effect, maintaining the high strength of the elastomers. Interestingly, although in pure elastomers the cavitation strength decreases with temperature, we find that in these filled elastomers the critical stress for the onset of cavitation increases significantly with temperature strongly suggesting that cavitation is due to the local confinement between fillers and supporting the idea of a glassy layer near the filler. Aging for 10 days at $110^{\circ} \mathrm{C}$ in oxygen-free conditions decreases both SIC and cavitation and reduces the strength of the elastomer at high temperature. This is attributed to the formation of sulfur side chains hindering the crystallization.
\end{abstract}




\section{Introduction}

Strain-induced Crystallization (SIC) in natural rubber is a fascinating property for polymer physicists and rubber technologists alike. Although natural rubber is not, by far, the only soft polymer able to crystallize under strain, its technological relevance, which far exceeds all other examples, justifies indepth studies on the origin of this phenomenon. Numerous investigations have been published over the years focusing on this peculiar property ${ }^{1,2}$. Most of the work focused on unfilled and lightly crosslinked natural rubber ${ }^{3-7}$, which is highly extensible before fracture occurs. Some studies were however extended to more realistic filled systems ${ }^{8-11}$, at room temperature and high temperature, and to the strain induced crystallization upon fast loading conditions ${ }^{12-14}$, but none took as benchmark the typical conditions and materials found in truck tyres in operation, i.e. a combination of high crosslink density obtained with a sulfur based conventional curing chemistry, highly reinforcing carbon black filler and high service temperature for a long time in the absence of oxydation.

Such conditions are found between steel plies of the carcass of a truck tyre. The need to bond to the steel cord imposes high levels of added sulfur and therefore a high crosslink density, while the position of the rubber inside the tyre results in non oxydizing aging conditions and a temperature of the order of $100^{\circ} \mathrm{C}$ for highway driving. The combination of high stress, high temperature and high confinement makes this spot a critical safety spot for the tyre and materials requirements are particularly stringent.

The main results of previous studies on strain-induced crystallization of natural rubber can be summarized as follows. SIC is a thermodynamically driven phenomenon triggered by a decrease in entropy of the polymer chains as they are stretched, which reduces the barrier to form crystals. Within that framework, the higher the deformation of the chains and the lower the temperature, and the more chains can crystallize along the stretching direction. This has been demonstrated in lightly crosslinked unfilled natural rubbers. However the effect of increasing the crosslink density is less obvious to interpret. For peroxide crosslinked natural rubber increasing the crosslink density clearly favours SIC in terms of onset of crystallinity and the crystallization "rate", i.e. the increase in crystallinity with increasing stretch $\lambda^{7,15}$. However for the much more technologically relevant case of sulfur crosslinking the situation is more complex. Some studies report no change in the onset of SIC with increasing sulfur content ${ }^{16}$ while others do report an increase in SIC with increasing density of crosslinks ${ }^{3}$. Chenal et al. have argued that if an effective density of crosslinks is extracted from swelling experiments (for filled and unfilled systems), there exists an optimum in crystallization "rate" for an intermediate density of crosslinks. All of these seemingly contradictory results suggest that the details of the crosslinked structure (homogeneous vs heterogeneous, presence or not of defects along the chains introduced by the crosslinking process) can all have an influence on the SIC process.

The incorporation of nanoparticles as filler, in addition to the well-known stiffening and toughening effects observed also in amorphous rubbers, also significantly lowers the threshold stretch at which SIC is observed. The simple explanation for this well-known effect is a strain amplification of the polymer chains due to the presence of filler ${ }^{8,17,18}$ but this explanation remains rather simplistic. The effect of temperature on SIC has also been demonstrated clearly ${ }^{3,10,19,20}$ but the focus was on unfilled systems and moderate temperatures.

Finally, recent studies using small-angle X-ray scattering have shown that when highly reinforcing filler particles are used in the material, nanocavities (30-50 nm in size) form at high stretch ratios causing an increase in volume ${ }^{18,21}$. Although these nanocavities are only observed during the first stretching cycle in uniaxial tension ${ }^{22}$, and are therefore associated to the damage processes often called Mullins effect, they have been observed also at the tip of a crack and may be relevant for its propagation ${ }^{23}$. 
The effect of thermal ageing on strain-induced crystallization in natural rubber has never been investigated to the best of our knowledge. A study by Le Gac showed that thermo-oxidative ageing caused a decrease of the strain induced crystallization in polychloroprene ${ }^{24}$. The effect of thermooxidative ageing on crystallization at a crack tip in natural rubber is also shown in the $\mathrm{PhD}$ of Rublon ${ }^{25}$, and the crystallized volumes at the crack tip were slightly smaller after ageing. However, no study focused on purely thermal aging of natural rubber.

In this study we report X-ray scattering experiments at small and large angle carried out with synchrotron radiation to investigate strain-induced crystallization and cavitation of model filled and unfilled naturals rubbers stretched in uniaxial tension. Unlike most previous studies our materials have been vulcanized conventionally with $6 \mathrm{phr}$ of sulfur, resulting in a high crosslink density $\left(\mathrm{M}_{\mathrm{c}}=9\right.$ $\mathrm{kg} / \mathrm{mole}$ ). The effect of two specific parameters on SIC and cavitation will be investigated, i.e. temperature and aging conditions in oxygen starved conditions.

\section{Materials and Methods}

Vulcanized NR samples were kindly provided by Michelin. The unvulcanized NR had a $\mathrm{M}_{\mathrm{w}}=1300$ $\mathrm{kg} / \mathrm{mol}$ and a PDI $=3.9$. The filler was CB N347 and was introduced into the rubber matrix in fractions varying between $0 \mathrm{phr}$ (parts of weight per hundred parts of rubber) and $70 \mathrm{phr}$, corresponding to volume fractions between 0 and $27 \%$, respectively. The density of the matrix and the filler are $\rho=0.92 \mathrm{~g} / \mathrm{cm}^{3}$ and $\rho=1.8 \mathrm{~g} / \mathrm{cm}^{3}$. The composition of the materials used is summarized in table 1 . Samples were prepared in the following way: all ingredients except vulcanizing agents were first mixed in a lab scale Haake internal mixer. The vulcanization agent was then incorporated in a calendering machine following a procedure developed by Michelin. The sheet was then cut into pieces into a mold and put in an oven at $150^{\circ} \mathrm{C}$ for vulcanization. The vulcanization time for each material was optimized with a rheological measurement at $150^{\circ} \mathrm{C}$. Finally, samples were removed from the molds, air-cooled and tensile dumbbell samples were punched. Samples had a $40 \mathrm{~mm}$ effective length and a $4 \mathrm{~mm}$ by $2-2.5 \mathrm{~mm}$ cross section.

\begin{tabular}{|c|c|c|c|c|c|c|}
\hline Material & NR0 & NR30 & NR50 & NR70 & NR50 S- & $\begin{array}{c}\text { NR50 } \\
\text { Rublon }\end{array}$ \\
\hline NR & 100 & 100 & 100 & 100 & 100 & 100 \\
\hline $\begin{array}{c}\text { Carbon black } \\
\text { (N347) }\end{array}$ & 0 & 30 & 50 & 70 & 50 & 50 \\
\hline $\begin{array}{c}\text { Carbon black } \\
\text { (vol\%) }\end{array}$ & 0 & 13.5 & 17.3 & 26.7 & 17.3 & 17.3 \\
\hline 6PPD & 2 & 2 & 2 & 2 & 2 & 1.9 \\
\hline Stearic Acid & 2 & 2 & 2 & 2 & 2 & 2 \\
\hline ZnO & 5 & 5 & 5 & 5 & 5 & 2.5 \\
\hline DCBS & 1 & 1 & 1 & 1 & 0.5 & 1.6 \\
\hline Sulfur & 6 & 6 & 6 & 6 & 3 & 1.6 \\
\hline$v\left(\mathrm{~mol} / \mathrm{cm}^{3}\right)$ & $2.5 \times 10^{-4}$ & $2.5 \times 10^{-4}$ & $2.5 \times 10^{-4}$ & $2.5 \times 10^{-4}$ & $1.5 \times 10^{-4}$ & $1.3 \times 10^{-4}$ \\
\hline $\mathrm{M}_{\mathrm{c}}(\mathrm{kg} / \mathrm{mol})$ & 9 & 9 & 9 & 9 & 15 & 17.4 \\
\hline
\end{tabular}

Table 1: Material composition. The reference material is highlighted in red, the modified values for the other materials in green.

For the ageing process the samples were placed in a nitrogen filled and sealed oven and kept for ten days at $110^{\circ} \mathrm{C}$. These samples will be referred to as A10 in the following.

The large strain mechanical properties were characterized in uniaxial tension until fracture on an Instron tensile testing apparatus. The samples were deformed at a constant stretch rate $\dot{\lambda}=2 \mathrm{~s}^{-1}$. For each material, three samples were tested to failure. 
The average molecular weight between crosslinks of the samples was determined by swelling experiments using a calibration procedure developed by Michelin. The values are reported in Table 1 in terms of density of crosslinks and average molecular weight between crosslinks. The average molecular weight between entanglements of poly cis 1,4 polyisoprene is $M_{e}=7 \mathrm{~kg} / \mathrm{mole}$.

\subsection{WAXS and SAXS}

Wide and Small angle X-ray scattering were used to characterize the degree of strain-induced crystallization and the occurrence of cavitation in the samples during uniaxial tension tests. All X-rays measurements were carried out with Synchrotron radiation on different beamlines. The beamlines used were selected based on their capability to perform both WAXS (for SIC detection) and SAXS (for cavitation detection). The lines used were BM02 at European Radiation Synchrotron Facility (Grenoble, France), 5ID-D at the Advanced Photon Source (Argonne, USA) (two runs), and SWING at SOLEIL (Saclay, France). The energy, wavelength, detector distances and q range used are listed in table 2.

\begin{tabular}{|l|l|l|l|l|l|l|}
\hline & $\begin{array}{l}\text { Energy } \\
(\mathrm{kEV})\end{array}$ & $\begin{array}{l}\text { Wavelength } \\
(\mathrm{nm})\end{array}$ & $\begin{array}{l}\text { WAXS } \\
\text { distance } \\
(\mathrm{m})\end{array}$ & $\begin{array}{l}\text { SAXS } \\
\text { distance } \\
(\mathrm{m})\end{array}$ & $\begin{array}{l}\text { WAXS } \\
2 \theta \\
\text { range } \\
\left(\mathrm{nm}^{-1}\right)\end{array}$ & $\begin{array}{l}\text { SAXS } \\
\mathrm{q} \\
\text { range } \\
\left(\mathrm{nm}^{-1}\right)\end{array}$ \\
\hline BM02 & 11 & 0.113 & 0.198 & 1.920 & $\begin{array}{l}4.75- \\
30\end{array}$ & $\begin{array}{l}0.020- \\
0.87\end{array}$ \\
\hline SWING & 8 & 0.155 & 0.198 & 6.279 & $\begin{array}{l}4.75- \\
30\end{array}$ & $\begin{array}{l}0.017- \\
0.78\end{array}$ \\
\hline $\begin{array}{l}\text { 5ID-D } \\
\text { (A) }\end{array}$ & 10 & 0.124 & 0.198 & 7.496 & $\begin{array}{l}4.75- \\
30\end{array}$ & $\begin{array}{l}0.020- \\
0.87\end{array}$ \\
\hline $\begin{array}{l}\text { 5ID-D } \\
\text { (B) }\end{array}$ & 9.8 & 0.127 & 0.200 & 8.505 & $4.5-30$ & $\begin{array}{l}0.013- \\
0.78\end{array}$ \\
\hline
\end{tabular}

Table 2 : Characteristics of the different beamlines used in this study.

Each run was designed to overlap and complement the previous one. The run at ESRF provided data on the crystallinity of fresh materials at different temperatures. The first run at APS provided results on both new and aged materials, at room temperature. The experiment conducted at SOLEIL and the second run at APS provided results on high temperature for both new and aged materials.

The specific set-ups used for the tensile measurements and the data acquisition details are provided in the supplementary information.

\subsubsection{Set-up used at 5ID-D}

5ID-D was equipped with two different tensile devices for the two runs (A and B). During run A, no-high temperature tests were planned and a simple Instron tensile device provided by the beamline staff was used.

A $12.5 \mathrm{~mm} / \mathrm{min}$ crosshead speed was used, for an initial strain rate of $5.10^{-3} \mathrm{~s}^{-1}$. Stress data was extracted from the Instron load cell, as well as crosshead position. Clamps had a flat design with a rough surface and slipping from the clamps was sometimes observed at high strain. Therefore, a direct optical strain measurement was performed. As shown in Figure S1, dots were painted with a white paint and video tracking of theses dots was used to compute the local strain in the center of the sample. Stress strain data were compared and matched the mechanical data obtained ex-situ for the same material (Figure S2).

Between run A and run B, the 5ID-D beamline acquired a Linkam TST350 device, capable of running symmetric tensile test at various temperatures. The Linkam, used in run $\mathrm{B}$, is composed of a tensile stage, enclosed in an airtight oven with a heating element designed to be in contact with the sample. The heating element contains a hole to let the x-ray beam through. 
While highly convenient for testing at high temperature, this stage had several drawbacks for the specific needs of the experiment that had to be addressed. The main one was due to the clamps geometry. The minimum distance between clamps was $26 \mathrm{~mm}$, with a maximum amplitude of displacement of $80 \mathrm{~mm}$, limiting the stretch ratio of the sample to $\lambda=3.1$, a much lower value than the measured failure stretch ratio of some of the materials tested. Moreover, the flat, smooth geometry of the clamp suggested that significant slippage of the sample would occur, and the Linkam design did not allow for optical measurement of strain.

To address these issues, new clamps were designed to fit a custom sample. This sample has a $6 \mathrm{~mm}$ high, $1 \mathrm{~mm}$ thick and 3-4mm wide effective gauge area. The top and bottom part of this area are extended by two horizontal cylinders, pinned in the clamps to prevent slippage. The new clamps had to be mounted on top of the existing support plates to preserve the integrity of the Linkam, and the sample was no longer directly in contact with the heating element. To ensure a proper control of the temperature, three thermocouples were installed (one in contact with the sample, one in the clamp, one in the oven far away from the clamps). The oven was filled with helium gas for a better thermal conductivity.

\subsubsection{Set up used at BMO2 and SWING}

Neither BM02 nor SWING had a dedicated tensile stage for in-situ X-ray experiments. A tensile stage, pictured on Figure S3, using two counter-rotating cylinders to extend the material in a symmetric fashion and a heating element was used in those experiments. Since the material is rolled around the cylinders, the central part of the sample, where the X-ray beam hits, can reach large stretch ratios in a confined space, a very useful property for in-situ testing ${ }^{26}$.

This tensile stage was however not equipped for high temperature testing, and a new oven had to be developed. The chosen design used conduction to heat the sample, as carbon black filled elastomers are good thermal conductors. Two thermally regulated cylindrical heating elements were inserted in a perforated brass cube designed to be in contact with the sample. A brass window, sealed by a kapton tape (transparent to X-rays) was used to close the hole on the other side of the sample to limit heat loss into air. The whole heating element was enclosed on the sides by insulating foam and a steel case, to prevent heating of the stage itself, which would have caused unwanted stresses due to thermal expansion of the gears. A photograph of actual mechanical set up used for the tensile experiment at high temperature is shown on Figure S4.

This oven was tested using thermocouples on the material, and IR-imaging (Figure S5). The heat proved to be quite homogeneous in the section of the material exposed to $\mathrm{X}$-rays, and the oven was very effective, allowing to heat the material up to $120^{\circ} \mathrm{C}$ for a temperature of $150^{\circ} \mathrm{C}$ applied on the heating elements.

\subsubsection{Values recorded during the experiment}

During an in-situ test, the stretching is continuous, while the data recording is discrete. To ensure proper processing of the scattering images $\mathrm{I}_{\text {acquired}}$, several mechanical measurements are recorded for each $\mathrm{X}$-ray pattern capture. The maximum image sampling frequency is dictated by the acquisition, readout and processing time of the beamline. A 5 to 10s gap was typically used between two acquisitions while the exposure time was set between 20 and $500 \mathrm{~ms}$.

The beam intensity before the sample was defined as IC1 and after the sample was defined as IC2. They were respectively measured (as photon counts) by an ion chamber and a pin diode. Since two different devices were used to measure theses intensities, a correlation factor $\mathrm{R}$ defined as $\mathrm{R}=\mathrm{IC} 1_{\text {air }} / \mathrm{IC} 2_{\text {air }}$ was used to renormalize the data. The scattering patterns of air alone $\mathrm{I}_{\text {air }}$ and with a zero beam intensity $\mathrm{I}_{\text {dark }}$ were also recorded for data correction purposes.

While all these data are synchronized with the detector exposure, measurement of stress, strain, or temperatures were often recorded on external devices. Synchronization was obtained using a simultaneous start on all devices, as well as timestamps associated with each data set. 


\subsubsection{WAXS analysis : crystallinity index}

While the absolute value of the degree of crystallinity cannot be determined, a variety of definitions for the crystallinity index exist in the literature. Three types of definitions for the crystallinity index have mainly been used. The first type uses the sole value of the intensity of the crystalline peaks ${ }^{27,28}$. The second focuses on the variation of the amorphous intensity ${ }^{8,29,30}$ while the last type computes a ratio between crystalline intensity and total diffracted intensity ${ }^{4,6,16,31-34}$. All of these indexes are based on the azimuthally integrated intensity of the 2D pattern in a given range from the equator. The resulting intensity profile is then in most cases deconvoluted into crystalline and amorphous peaks, using Gaussian or Pearson functions, the parameters of the deconvolution being then used to compute the crystallinity index.

In this study, the wide range of testing conditions of the various WAXS experiments showed that this deconvolution was not always possible. At low levels of crystallinity, the signal to noise ratio is sometimes too low to carry a proper deconvolution, so a different technique was used.

First, the diffraction intensity obtained from the WAXS detector was azimuthally integrated in the range of $-5 / 5^{\circ}$ from the equator (see Figure S6 and Figure S7 of the supplementary information). The choice of a limited azimuth angle for the crystalline signal was made to improve the signal to noise ratio. This total signal contains both crystalline peaks 120 and 200, and the amorphous halo. The amorphous contribution is then estimated using either a fit on the amorphous signal outside the peaks, or with a second integration in the range of $15^{\circ} / 25^{\circ}$ from the equator (green lines in Figure S6). As the amorphous halo is slightly dependant on the angle to the equator, this range was chosen to be the closest range that did not intersect with the crystalline peaks. Finally, the contribution of crystallinity to the scattered intensity is obtained by subtracting the amorphous contribution from the total signal. Examples of the integration sectors and resulting intensity profiles for a stretched unfilled natural rubber are given in Figure S6 and Figure S7. To ensure a consistent value of the index through the various X-rays experiments, these signals were integrated over a constant Bragg range of $2 \theta=9.9^{\circ} / 19.5^{\circ}$, and the final index was defined as:

$$
\chi_{S I C}=\int_{2 \theta} \frac{I_{c r}}{I_{a m}+I_{c r}}
$$

\subsubsection{SAXS analysis: void volume fraction}

Nanocavities can be detected in the material from the variation of the scattering invariant $Q$ following a procedure described in detail in a previous paper ${ }^{35}$. We recall here the main steps in data processing. The calculation of the scattering invariant to detect nanocavities requires the proper processing of the raw SAXS patterns. The acquired scattering pattern results from the scattering of both the sample and the air surrounding the sample in the space where the beam is outside the vacuum tubes. This scattering from air, as well as the zero intensity noise on the detector (dark values) needs to be corrected using the following formula:

$I_{\text {cor }}=I_{\text {raw }} \frac{R I C 2}{I C 1} \frac{I C 1}{I C 1_{\text {air }}}\left(I_{\text {air }}-I_{\text {dark }}\right)-I_{\text {dark }}$

where $\mathrm{I}_{\text {cor }}$ represents the scattering of the sample alone, IC1 and IC2 are the incident and transmitted intensity, $\mathrm{IC}_{\text {air }}$ is the incident intensity for the experiment done in the absence of the sample, $\mathrm{I}_{\text {air }}$ is the 2D scattering pattern for the same experiment and $\mathrm{I}_{\mathrm{dark}}$ is the image detected when the shutter is closed. Scattering is also dependent on variations in incident beam intensity IC1, sample thickness $\mu \mathrm{Ln}(\mathrm{RIC} 1 / \mathrm{IC} 2)$ and transmission $\mathrm{R}(\mathrm{IC} 1 / \mathrm{IC} 2)$ and $\mathrm{I}_{\text {cor }}$ needs to be corrected for these values ( $\mu$ is the absorption coefficient of the sample). However, since in our study all scatterings patterns and invariant values are compared to the values in the undeformed state $\left(I_{0}\right.$ and $\left.Q_{0}\right)$, the final corrected intensity $I_{\text {cor2 }}$ can be written as:

$$
I_{\text {cor } 2}=\frac{I_{\text {cor }}}{I C 2} \ln \left(\frac{I C 1}{R I C 2}\right)
$$


Examples of these corrected 2D scattering patterns at different values of $\lambda$ for the NR50 material are shown in Figure S8 of the supplementary information. Since the differences between the stretched and the unstretched state are not obvious in SAXS we also show the patterns normalized by the pattern obtained in the undeformed state which are used to calculate the relative invariant $\mathrm{Q} / \mathrm{Q}_{0}$.

The scattering invariant $\mathrm{Q}$ is the integral of this corrected intensity $\mathrm{I}_{\text {cor2 }}$ over all reciprocal space. With the assumption of cylindrical symmetry of the system along the tensile direction (x axis), it is expressed as follows ${ }^{35}$ :

$Q=\frac{1}{2} \int_{-\infty}^{+\infty} \int_{0}^{\infty} I_{\operatorname{cor} 2}\left(q_{x}, q_{y}\right) q_{y} d q_{x} d q_{y}$

The Q value is independent of the position of the scattering objects. Only newly created scattering objects, such as nano-cavities cause an increase in the scattering invariant. For non-crystallizable rubbers, a three phase (elastomer, filler, cavities) model was developed by Zhang et al ${ }^{35}$ and they established that the relative variation of Q was proportional to the volume fraction of cavities $\phi_{\text {void }}$ created:

$\frac{Q}{Q_{0}}-1=\left[\frac{\phi_{\mathrm{R}} \rho_{\mathrm{R}}^{2}+\phi_{\mathrm{CB}} \rho_{\mathrm{CB}}^{2}}{\phi_{\mathrm{R}} \phi_{\mathrm{CB}}\left(\rho_{\mathrm{R}}-\rho_{\mathrm{CB}}\right)^{2}}-1\right] \phi_{\text {void }}$

where $\phi_{\mathrm{CB}}, \phi_{\mathrm{R}}$ are the respective initial volume fractions of carbon black filler and rubber and $\rho_{\mathrm{CB}}$ and $\rho_{\mathrm{R}}$ their respective $\mathrm{X}$-ray scattering length densities $\left(X_{\mathrm{LSD}}\right)$. It should be noted that natural rubber contains also impurities such as proteins that contribute to the scattering of the material. The previous model was corrected $^{18}$ to account for this extra source of scattering. Assuming that the contribution of the impurities to the scattering invariant is unchanged with stretching, and neglecting second order interactions (such as filler/impurities), the correction is made by subtracting the value of the scattering invariant for an unfilled material, $Q_{u}$ :

$$
\left(\frac{Q}{Q_{0}}\right)_{c o r r}=\frac{Q-Q_{u}}{Q_{0}-Q_{u}}
$$

In our work, the value of $\mathrm{Q}_{\mathrm{u}}$ accounts only for a small fraction of $Q$ for filled materials, and, since unfilled materials were not available for all experiments, this correction was neglected.

While the theoretical value of $Q$ is calculated over an infinite $q$ range, actual experiments are limited to the range of $q$ available at the beamline. At small $q$, information is lost under the beamstop, while at high $q$, the scattered intensity quickly decays until it cannot be separated from the noise of the detector. Increasing the exposure time can extend the maximal observable $q$, but is limited by the saturation of the detector in the low $q$ region. To exploit an optimal range on the detector, two successful images were taken for each acquisition point. A first scattering pattern was recorded with a short exposure time $(t=0.02 \mathrm{~s}$ at APS $)$ and a small beamstop, and a second pattern was acquired with a longer exposure time $(t=0.5 \mathrm{~s})$ and a larger mask inserted to protect the low $q$ region of the detector. Both images are then merged using the mean intensity at a common $q_{\text {merge }}$ value $\left(q_{\text {merge }}=0.48 \mathrm{~nm}^{-1}\right)$.

Yet, observable $q$ values and respective real-space lengths remain limited both by the beamstop size and detector size, and this method leaves bigger and smaller cavities undetected. While information under the beamstop cannot be extrapolated, the calculation of the invariant can be carried beyond the maximum achievable $q$, using a Porod law to extrapolate the signal in the high $q$ region $^{22}$. Though this study showed that for high $q$, the actual choice of the cut-off $q_{\text {cut }}$ has a low impact on the Q calculation, we used a constant cutoff $q_{\text {cut }}=0.6 \mathrm{~nm}-1$ above which the scattering is assumed to follow Porod's law. 


\section{Results}

\subsection{Effect of filler content and degree of crosslinking}

The stress-strain curves for the unaged materials with different filler contents and different crosslink densities are shown in Figure 1. Compared to previously published studies, stress and strain at break are relatively low for these natural rubber samples, due to their high density of crosslinks. The effect of the presence of filler on the stiffness is clearly visible on Figure 1 and an optimum in strain at break is observed for $50 \mathrm{phr}$ of CB filler.
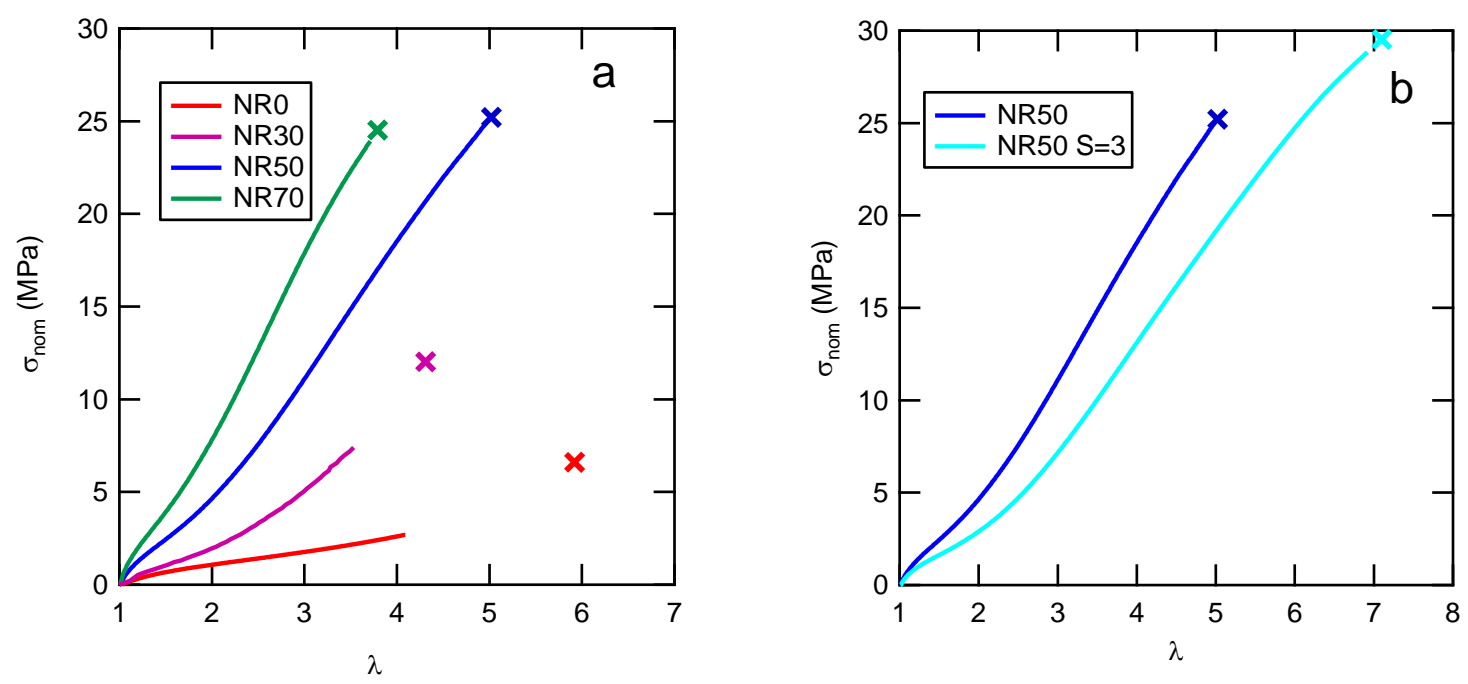

Figure 1: Effect of filler (a) and crosslinker content (b) on the stress vs stretch curves for different $C B$ content, at room temperature $\left(23^{\circ} \mathrm{C}\right)$ Each curve is the averaged value of 3 tests, up to the stretch ratio where the lowest breaking point is achieved. The cross represents the average breaking point.

Numerous previous investigations have shown that at room temperature $\left(23^{\circ} \mathrm{C}\right)$ and for unfilled natural rubber, strain induced crystallization (SIC) appears for a stretch ratio around $\lambda=4 .^{2,3,7,8}$ The degree of crystallinity then increases with stretch ratio up to the failure of the material. If a nanofiller is added, the onset of SIC in uniaxial tension generally shifts to lower values of $\lambda .^{2}$ This is confirmed for our highly crosslinked NR as shown on Figure 2a. This result can be rationalized by assuming that the presence of filler amplifies the local stretch seen by the polymer for the same macroscopic stretch ${ }^{8}$. A simple physical model of that strain amplification was developed by Bueche ${ }^{36}$ and Nielsen ${ }^{17}$ who proposed to use a corrected, local stretch ratio depending on the macroscopic extension ratio $\lambda$ and the filler volume fraction $\phi$ as:

$\lambda^{*}=1+\frac{\lambda-1}{1-\Phi^{1 / 3}}$

The measured and the corrected onset of crystallization $\lambda_{\text {SIC }}$ are plotted as a function of the filler volume fraction in Figure 2b. 

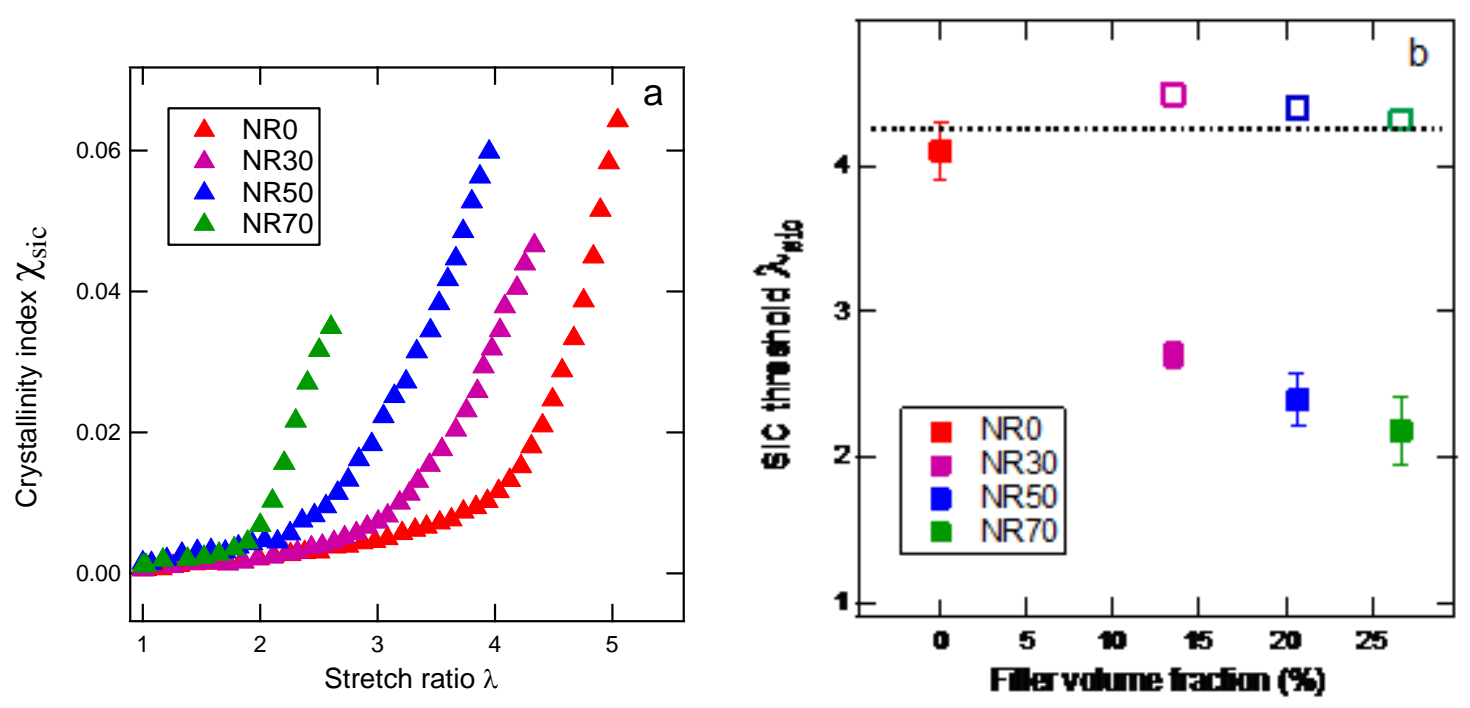

Figure 2:a) crystallinity index versus stretch ratio for materials with various amounts of filler. The slight slope before crystallization threshold is due to the created anisotropy in the amorphous phase before crystallization. $b$ ) stretch ratio at the SIC threshold as a function of filler volume fraction. The filled symbols are the measured values, the open symbols the corrected value calculated using the Nielsen formula

As discussed in the introduction the variation of crystallinity with crosslink density is complex and depends on the nature of the crosslinks and the presence or not of fillers and no consistent explanation has been proposed. Figure 3 shows the onset of SIC with $\lambda$ for three materials with the same filler content but a different crosslinking density: the reference NR50 $\left(\mathrm{M}_{\mathrm{c}}=9 \mathrm{~kg} / \mathrm{mole}\right)$, the reduced crosslinking NR50 $\left(\mathrm{M}_{\mathrm{c}}=15 \mathrm{~kg} / \mathrm{mole}\right)$ and an NR50 used in previous studies by Rublon and Zhang ${ }^{18,37}\left(\mathrm{M}_{\mathrm{c}}=17.4 \mathrm{~kg} / \mathrm{mole}\right)$. All of these materials have the same crystallization threshold despite a clear difference in crosslink density as shown in Table I, a characteristic that was observed in previous studies $^{3,7}$, but the increase in crystallinity after the threshold is different. A possible explanation can come from the nature of the crosslinking chemistry itself. The reference material $(9 \mathrm{~kg} / \mathrm{mole})$ and the less crosslinked material ( $15 \mathrm{~kg} / \mathrm{mole}$ ) have an unusually high crosslink density, and a very specific sulphur/accelerator (DCBS) ratio. Most of the materials previously studied were formulated using a 1/1 ratio, compared to the $6 / 1$ ratio used here. The structure of the crosslinks themselves (length of sulphur bonds) and the out of network sulphur could both affect the crystallization behaviour of these materials.

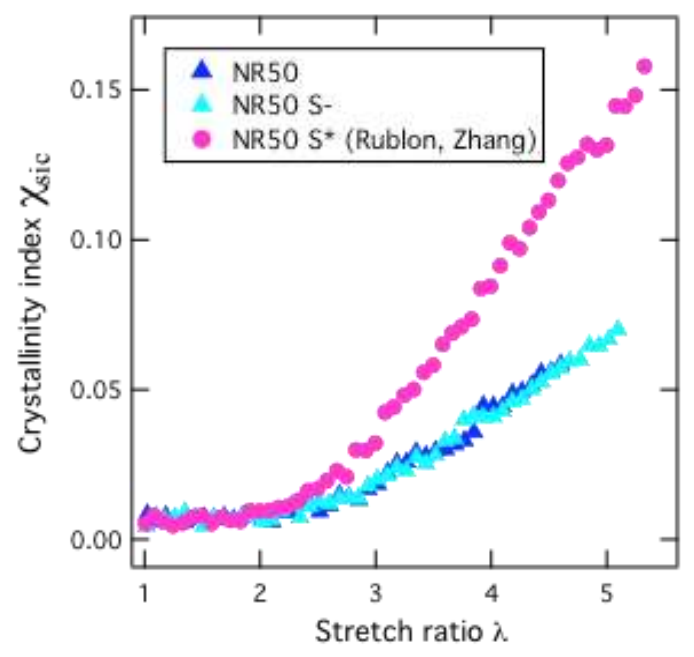


Figure 3: crystallinity index versus stretch ratio for NR50 with various amounts of sulphur. The $S=1.6$ NR50 is the same material that was tested in ${ }^{37}$.

As discussed in the introduction and in the materials and methods section, if nano-cavitation occurs when the material is stretched, it can be detected by the increase of the scattering invariant $Q / \mathrm{Q}_{0}{ }^{35}$ Previous work by Zhang \& al on less crosslinked NR but with the same filler, showed that the presence of filler was necessary to induce cavitation, and that the strain threshold for cavitation was reduced with increasing amount of filler. ${ }^{18}$

Figure 4 shows the variation of the scattering invariant versus stretch ratio. Cavitation was not detected before failure in the unfilled rubber or in NR30. However, for NR50 and NR70 a clear increase in scattering invariant was observed before macroscopic failure occurred. As discussed by Zhang et al. ${ }^{18,22,35}$, cavitation only occurs in filled rubbers because the filler locally creates hydrostatic stresses due to geometrical confinement. This local degree of confinement of the rubber between filler particles requires a high concentration of filler, and the macroscopic stretch needed to achieve it, decreases with increasing filler content.
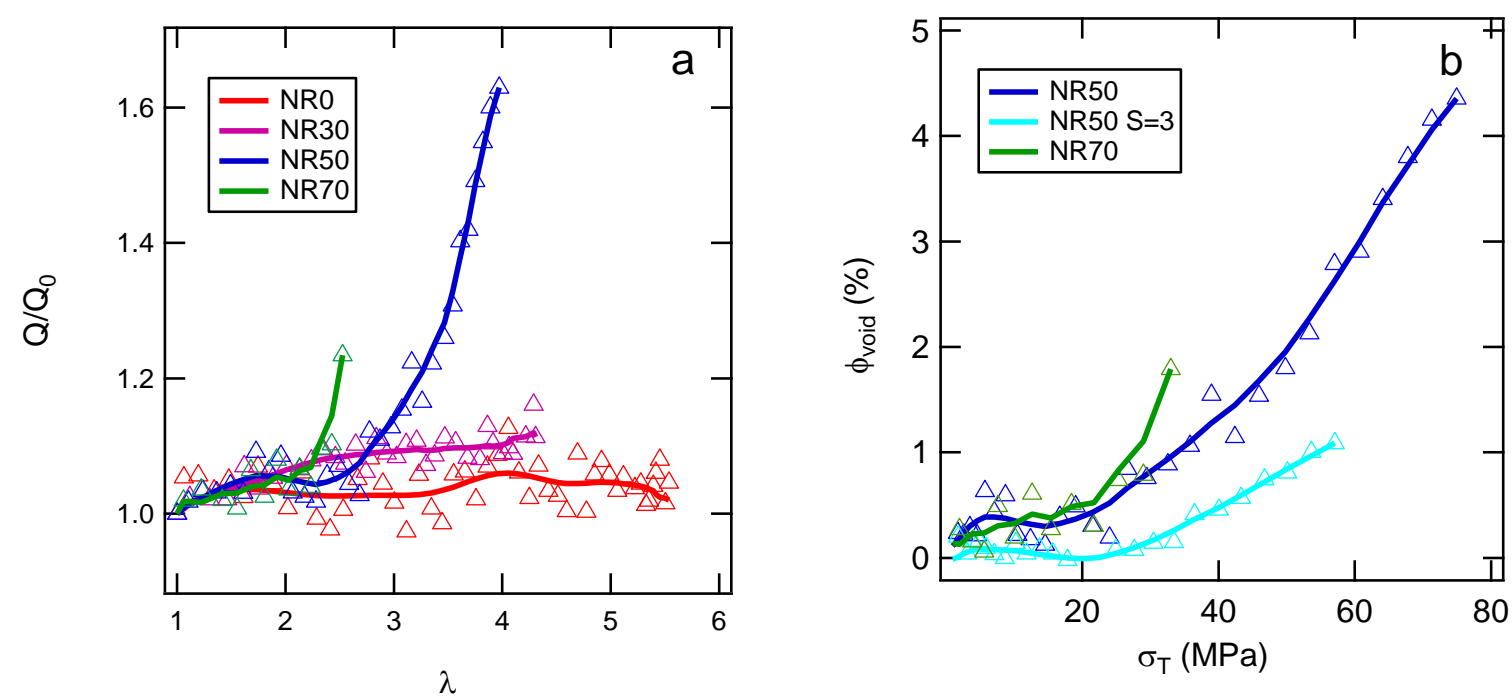

Figure 4: a) variation of the scattering invariant versus the stretch ratio, for materials with different amount of filler. $b$ ) Volume fraction of voids as a function of stress for the three materials were voids were observed before fracture. Markers are experimental values, and plain lines are smoothened values used as guides to the eyes.

While the good agreement of the Nielsen model for crystallization in filled rubbers implies that local strain dominates the crystallization behavior, previous work suggests that local hydrostatic stress triggers cavitation. ${ }^{18,35}$ Figure $4 \mathrm{~b}$ shows the volume fraction of voids obtained from equation 5 for all the cavitating materials as a function of true stress. All three materials appear to have the same true stress threshold of roughly $25 \mathrm{MPa}$, a very similar value as that found by Zhang \& al for filled and noncrystallizing Styrene-butadiene rubbers (SBR) ${ }^{35}$ However the cavitation threshold does not depend on filler volume fraction, unlike what was observed by Zhang $\&$ al in less crosslinked filled natural rubbers. ${ }^{18}$ This discrepancy is not surprising. As the threshold for crystallization depends on strain, and the threshold for cavitation on stress, more crosslinked materials crystallize much less and the crystallinity level at the cavitation threshold is negligible. Furthermore, cavitation occurs in the highly confined rubber near the filler, while SIC must occur in the highly stretched free rubber. Therefore there is no obvious reason that would justify a great impact of strain induced crystallization on the local cavitation stress at threshold for these highly crosslinked NR.

\subsection{Effect of temperature}


Since a complete characterization of the materials at all temperatures is impossible to achieve, a subset of temperatures had to be used. Two of them appear to be most relevant: room temperature (easiest to test, and representing the rest state of the tire), and $100^{\circ} \mathrm{C}$ (average core temperature of truck tyres on highways). These two temperatures were used as a reference in this study.

Figure 5a shows the effect of a temperature change on the tensile properties, for both the reference material (NR50) and the unfilled material (NR0). The effect differs greatly for the two cases. The unfilled material appears stiffer at high temperature, in agreement with rubber elasticity theory, but suffers from a severe drop in both failure stress and stretch ratio. The reference material is unaffected by temperature at low strain, but softens significantly at high strain. As a consequence, while the failure stretch ratio sees little change with temperature, the failure stress drops (from 25 to $16 \mathrm{MPa}$ ). That behavior is the same for all the filled materials in this study.
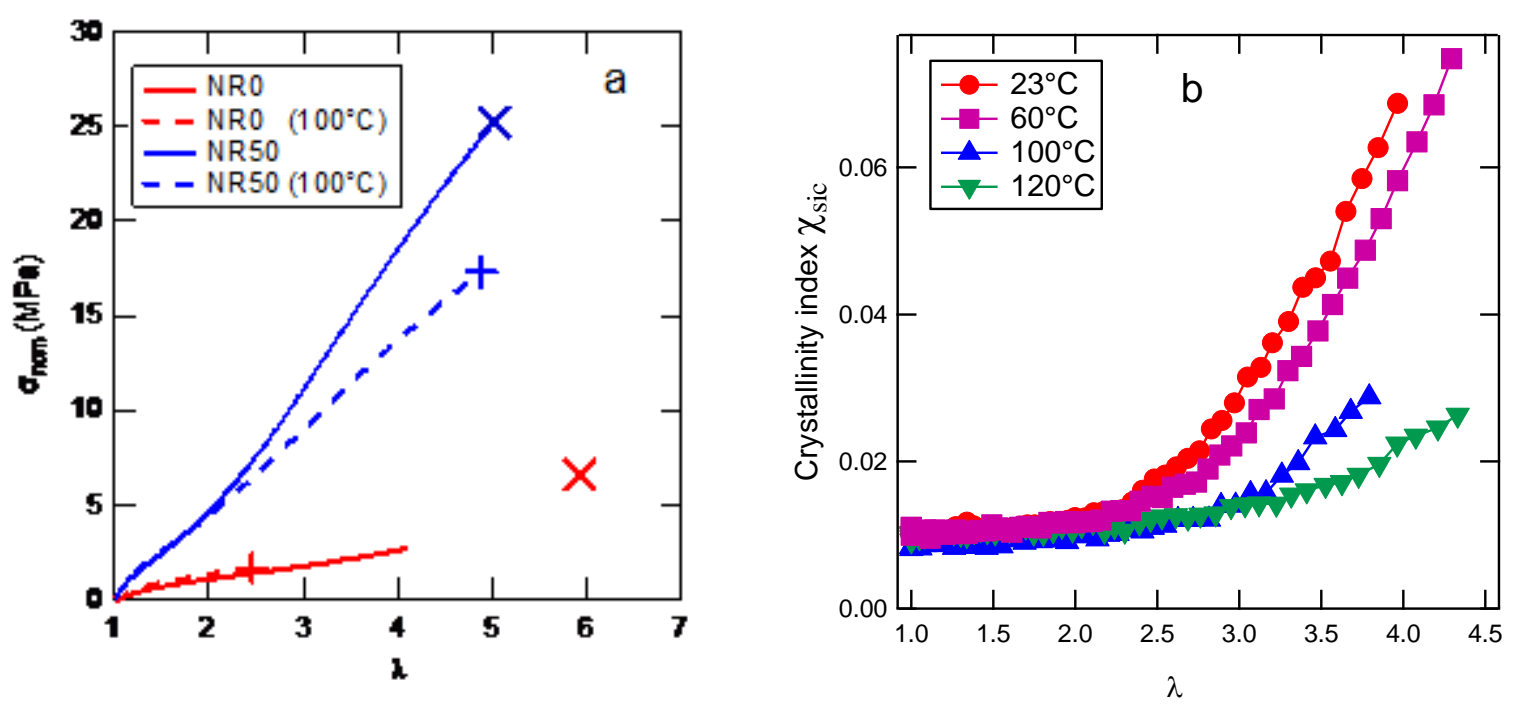

Figure 5: a) Stretch vs stress curves taken at $23^{\circ} \mathrm{C}$ and $100^{\circ} \mathrm{C}$ for NRO and NR50, using a $2 s^{-1}$ stretch rate. Each curve is the average value of 3 tests, up to the stretch ratio where the lowest breaking point is achieved. The cross represents the average breaking point. Full lines are data at $23^{\circ} \mathrm{C}$ and dashed lines are for $100^{\circ} \mathrm{C} \mathrm{b}$ ) Crystallinity index as a function of stretch at different temperatures for the NR50 material

Room and high temperature curves break apart at the stretch where the strain hardening appears at room temperature. Since the limiting extensibility of the chains does not change with $\mathrm{T}$, this difference is most likely due to a temperature sensitive reinforcing mechanism, such as SIC. Figure 5b shows the crystallinity index as a function of stretch ratio for the reference NR50, stretched at different test temperatures. Crystallinity appears at higher extension ratios for higher test temperatures, as observed in previous studies $^{2,3}$. However and surprisingly to us this highly crosslinked and filled rubber, SIC can still crystallize under stretch well before failure at a temperature as high as $120^{\circ} \mathrm{C}$ while previous studies report that unfilled well-crosslinked NR fully melts at much lower temperatures of the order of $70-90^{\circ} \mathrm{C}^{3,14}$.

Figure 6 summarizes the measured threshold stretches where the onset of SIC is observed as a function of temperature, for materials with various filler contents. As previously observed for less crosslinked natural rubber ${ }^{10,20}$, all of the crystallization thresholds increase with $T$. The overall linearity of the threshold value with temperature is verified for NR50. It should be noted that none of the other materials showed crystallization before failure at the highest temperatures. 

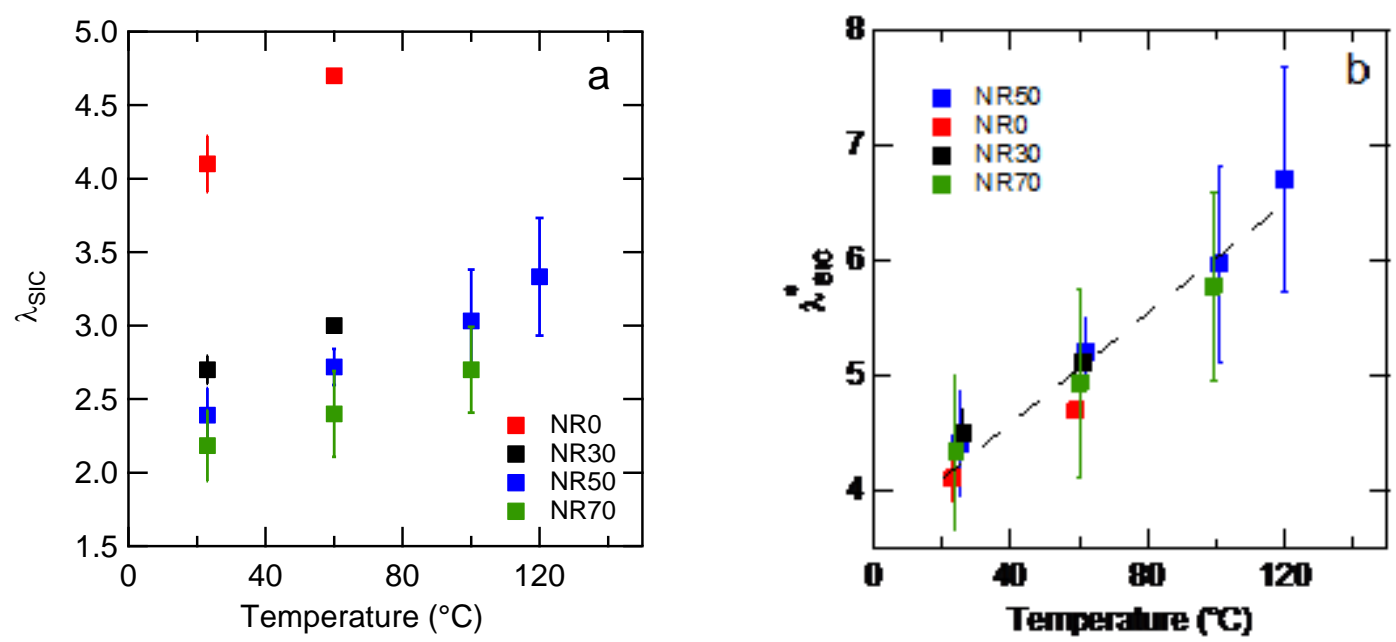

Figure $6: a)$ Values of $\lambda$ at the onset of crystallization $\left(\lambda_{\text {SIC }}\right)$ b) values of $\lambda_{\text {corr }}$ at the onset of SIC $\left(\lambda_{\text {SIC }}^{*}\right)$ plotted as a function of temperature.

If the threshold values are represented in terms of $\lambda_{\text {corr }}$ as shown on Figure $6 \mathrm{~b}$, Nielsen's model seems to work surprisingly well at high temperature, since all the materials start crystallizing at the same corrected extension ratio $\lambda_{\text {corr }}$ for a given temperature. The Nielsen model for the strain amplification due to the presence of filler seems therefore to be valid over the whole range of temperatures tested although error bars are large. The limitations of this new result are however the same as those demonstrated at room temperature: the model is unable to account for the differences in the rate of increase in crystallization with $\lambda$ after the threshold.

Unlike the effect of the amount of filler on SIC, no previous study measured the effect of crosslink density on the SIC during single stretch experiments at various temperatures. The thresholds for straininduced crystallization of the three differently crosslinked materials (NR50, NR50 S-, NR50 S--) are shown on Figure 7 and they appear to match at all temperatures.

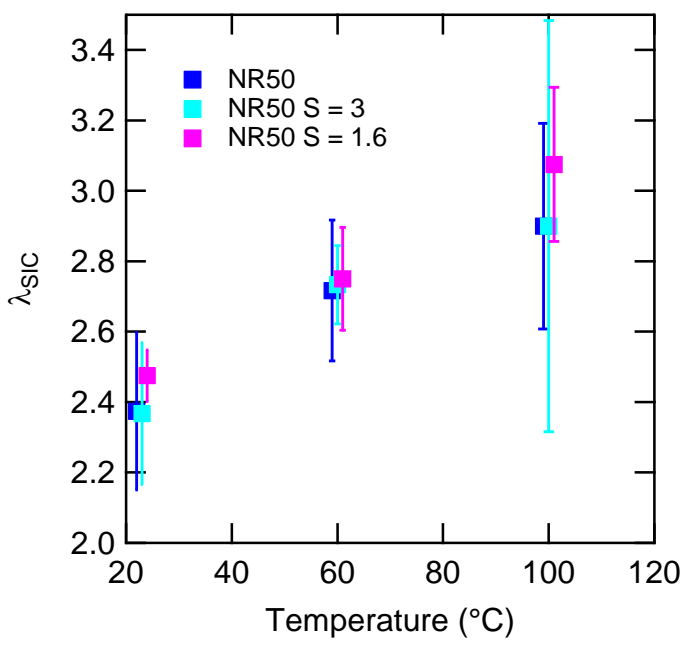

Figure 7 : Threshold stretch for the onset of SIC as a function of temperature.

In conclusion increasing the temperature decreases the SIC threshold regardless of the material, but the presence of filler may act as a nucleating agent for SIC, in a way that is qualitatively consistent with the Nielsen's model and has been noted for clay-filled natural rubber ${ }^{38}$. This certainly suggests that the carbon black may play a dual role as nucleating agent and also as toughening agent for the amorphous 
elastomer delaying crack propagation to levels of stretch where SIC can be activated and further toughen the material. Nevertheless it is remarkable that in these highly crosslinked materials, SIC is still observed before fracture at $120^{\circ} \mathrm{C}$ for the NR50 material while unfilled NR does not crystallize before fracture above $70^{\circ} \mathrm{C}$. This clear result has important implications justifying the use of filler to enhance high temperature toughness in NR.

\subsubsection{Effect of temperature on cavitation}

Unlike for SIC, all pre-existing studies of nano-cavitation in filled rubbers were made at room temperature. The results in this section constitute a fully new addition to the field.

Figure 8a shows the volume fraction of voids of the NR50 S- $(\mathrm{S}=3)$ as a function of stretch for three different test temperatures. Cavitation clearly appears at higher stretch ratios at higher temperatures. A similar behavior was observed for all other materials that showed cavitation at room temperature, except for the reference NR50 (S=6) which broke before cavitation was observed at $100^{\circ} \mathrm{C}$. Figure $8 \mathrm{~b}$ summarizes the measured threshold stretch ratio for all the tested materials and the increase in stretch ratio with temperature appears consistently for all of them. Figure 4 showed that the relevant parameter for cavitation is stress. As direct measurements of stress were not possible during in-situ tests at high temperature, the stretch-stress curves from Figure 1 and Figure 5, as well as the PHD thesis of Rublon ${ }^{25}$ were used to compute the stress at threshold for each material. The results are plotted on Figure 9. As the $60^{\circ} \mathrm{C}$ stress curves of the materials are missing, the value of the threshold were interpolated from the $23^{\circ} \mathrm{C}$ and $100^{\circ} \mathrm{C}$ stress-strain curves. Interestingly all this set of materials demonstrate the same stress at the cavitation threshold that clearly increases with temperature. This result can appear surprising, since previous studies ${ }^{39}$ carried out in pure elastomers under nearly hydrostatic loading showed a decreasing cavitation stress with increasing temperature (attributed to a drop in fracture energy $\Gamma$ ).

A tentative explanation for this discrepancy is the condition necessary to create local hydrostatic stresses in the material. The measured threshold stresses are indeed macroscopic tensile stresses. The hydrostatic stresses responsible for cavitation are local stress fields created by the confinement of the elastomer near the clusters of the filler particles. A proposed schematic view of this confinement is shown in Figure 10. At room temperature, the elastomer immediately embedding the filler (in blue) has a reduced mobility, and, according to the glassy layer mode $\mathrm{l}^{40}$ proposed by Lequeux and coworkers, a high apparent stiffness. This creates a percolating stiff network of glassy polymer and filler. This network is responsible for the local confinement of rubber volumes, and thus creates the required hydrostatic stresses to cause nanocavitation. As the temperature increases, the hydrostatic stresses required to nucleate cavities in the elastomers decrease. However, the glassy bridges between the rafts of filler are softened by the higher temperature, and the overall network gains mobility, reducing the level of local confinement and therefore the level of hydrostatic stresses generated by the same macroscopic average stress. This proposed mechanism would explain why the increasing tensile stress required for cavitation increases with temperature. It's a competition between the reduced degree of confinement, and the reduced fracture energy, and the variation in confinement seems to dominate, which is an interesting result. 

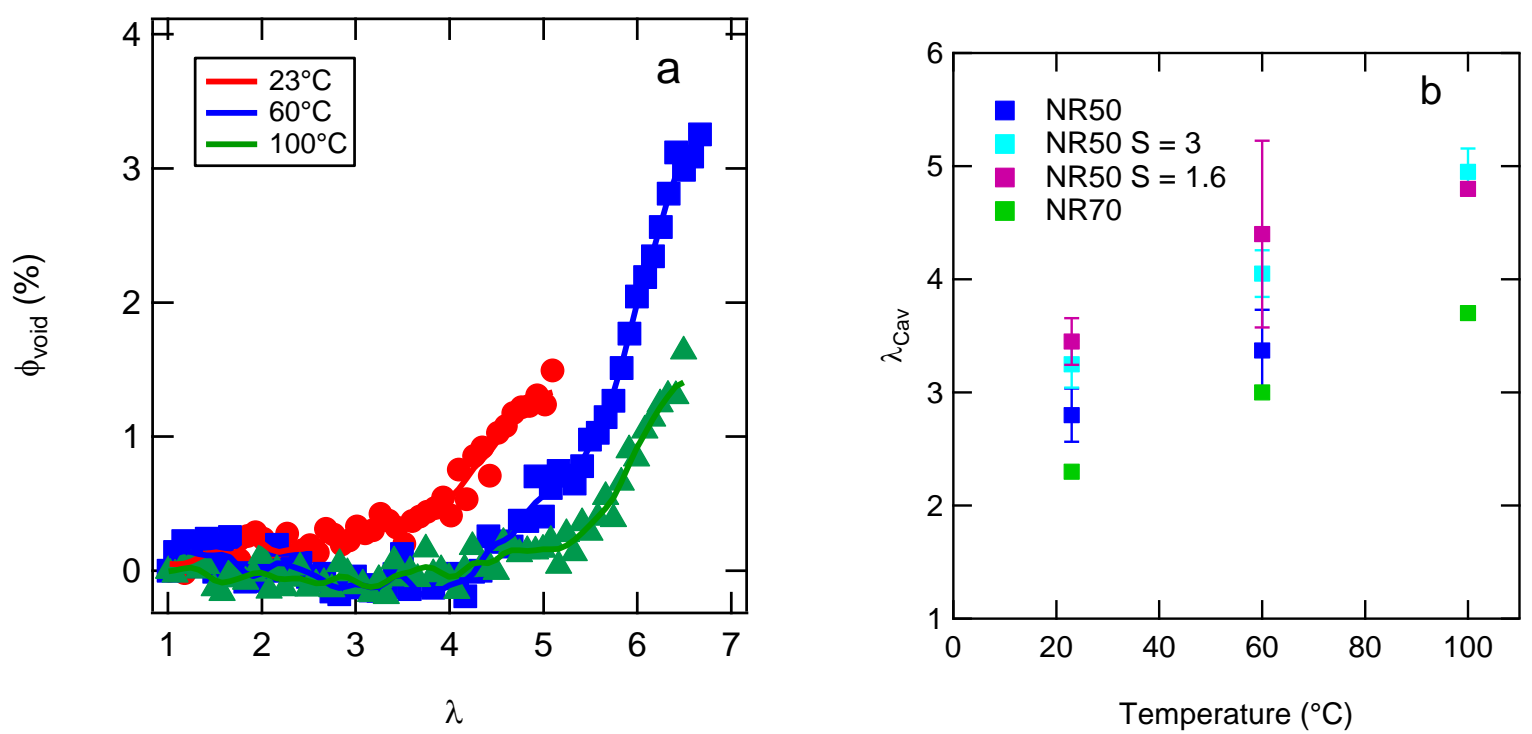

Figure 8: a) variation of the volume fraction of voids versus the stretch ratio for the NR50 S- $(S=3)$ tested at three different temperatures. Plain lines are smoothened values used as guide to the eyes. $b$ ) stretch ratio at cavitation threshold versus the test temperature for all the cavitating materials

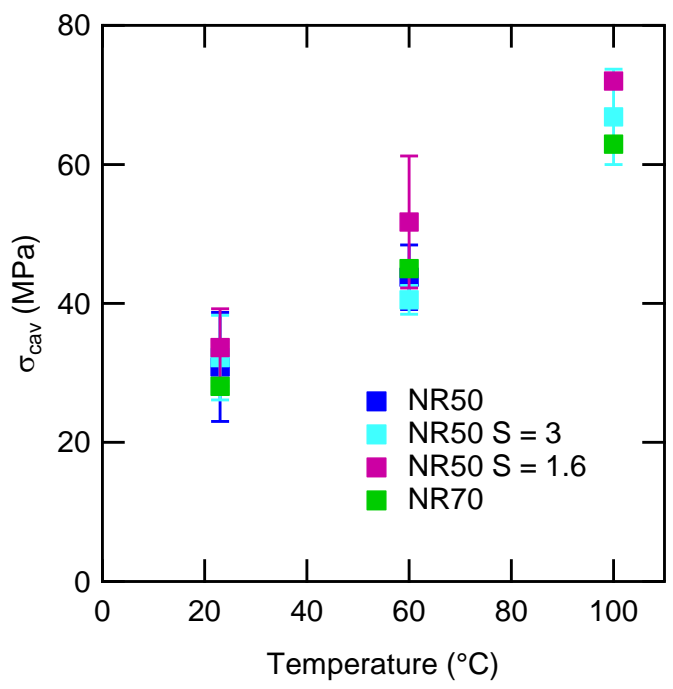

Figure 9: true stress at cavitation threshold versus temperature for all the cavitating materials.
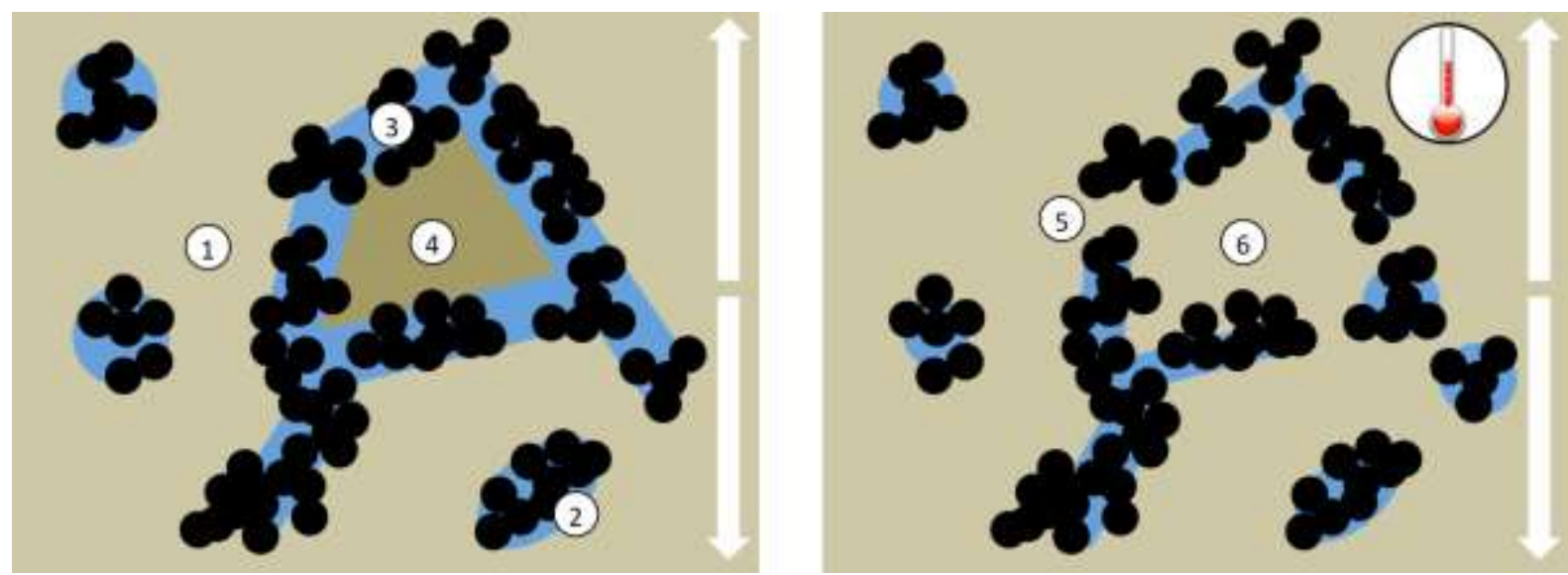

Figure 10: proposed mechanism for hydrostatic stress release in the materials. Tensile direction is vertical. Left: room temperature. Right: high temperature. (1): free elastomer, (2): isolated filler 
cluster, (3): percolating filler network, (4): confined elastomer, (5): depercolation in filler network, (6): freed elastomer.

\subsection{Effect of Ageing}

\subsubsection{Ageing and SIC}

As discussed in the introduction, the effects of thermal ageing on natural rubber are not well documented. As shown on Figure 11 the changes in uniaxial tensile mechanical behaviour remain subtle between the pristine materials and the materials aged at $110^{\circ} \mathrm{C}$ for 10 days in oxygen free conditions. At room temperature (Figure 11a), aged materials appear slightly softer. The unfilled material also gains additional stretchability. These two changes suggest a reduced crosslink density in the material after ageing. At $100^{\circ} \mathrm{C}$, a clear difference appears between filled and unfilled material after ageing. The unfilled material is nearly unaffected by ageing while remaining slightly softer. However, filled materials such as NR50 suffer from a large drop in failure stretch ratios.
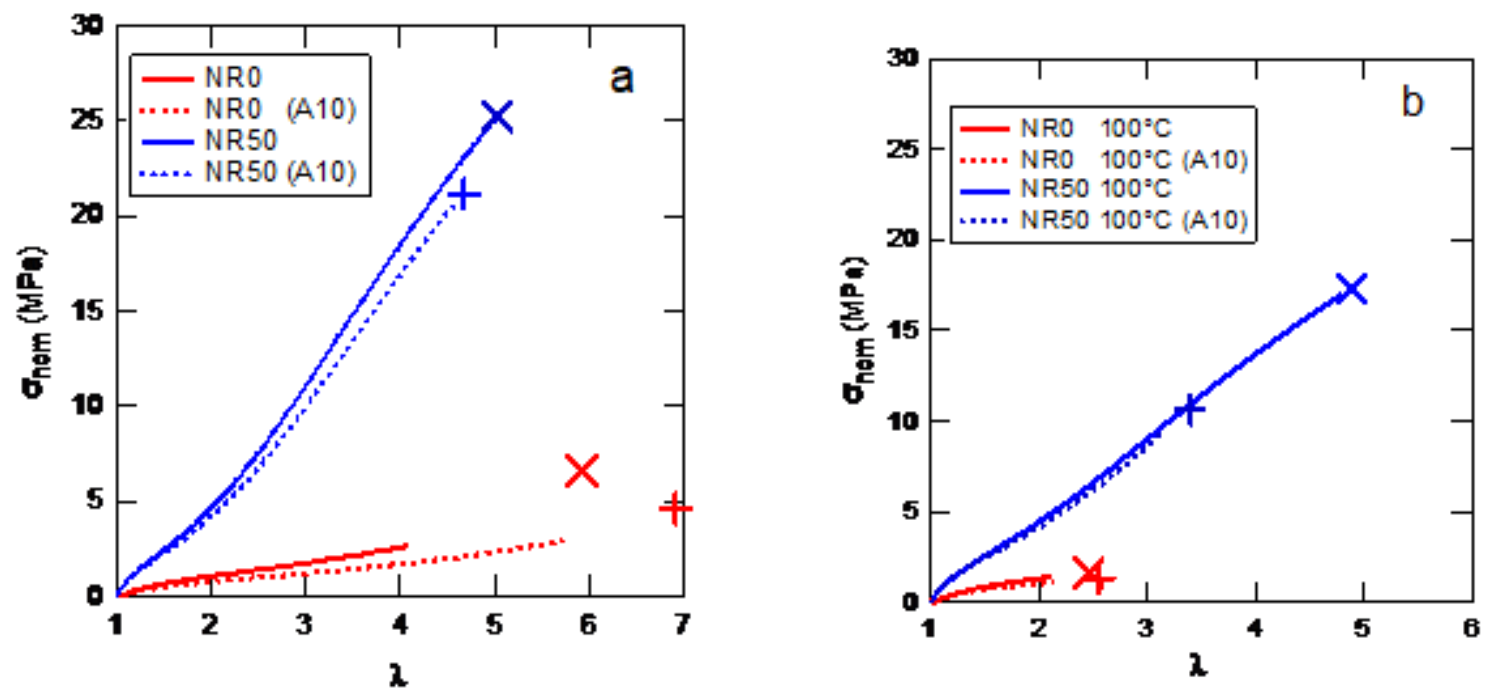

Figure 11: Stress vs stretch curves of new and aged (A10) NRO and NR50. a) at $23^{\circ} \mathrm{C}$ and b) at $100^{\circ} \mathrm{C}$ using a $2 s^{-1}$ stretch rate. Each curve is the averaged value of 3 tests, up to the stretch ratio where the lowest breaking point is achieved. Full lines represent pristine materials while the dashed lines represent the aged materials. The cross represents the averaged breaking point.

Little can be said at this stage on the reasons behind these differences, yet a few observations can be made. The "fresh" NR0 was already strongly weakened at high temperature, and ageing did not affect it further. This is consistent with the hypothesis of an absence of SIC before fracture at $100^{\circ} \mathrm{C}$ in unfilled NR, whether new or aged. However as shown in Figure $5 b$ pristine NR50 can crystallize under strain even at $100^{\circ} \mathrm{C}$. The reason for the drop in stretch at break after ageing could in principle be due to a reduced presence or even a complete absence of SIC due to ageing. Indeed the aged materials can still crystallize when stretched, but the crystallization thresholds, shown in Figure 12, are shifted to significantly higher stretches than for pristine materials. The initial crosslink density does not impact the shift in crystallization threshold of the material after ageing. However the effect of ageing on the crystallization threshold seems to decrease in the presence of filler, since the gap between the threshold of new and aged materials drops with the increase in filler volume fraction.

The results presented here address somewhat unusual materials studied on an undocumented area and as such are worth a more detailed discussion. The reason of the decrease in crystallinity itself is not 
obvious. The uniaxial tensile data in Figure 11 shows that the crosslink density was slightly reduced with aging (lower modulus). Considering the results of Figure 7 this should either not affect crystallization, or be slightly beneficial. The change in crosslink density therefore does not seem to be the dominant mechanism to explain the reduced crystallinity. Two hypotheses can therefore be formulated to explain this delayed crystallization:

1) In sulphur crosslinked elastomers, the nature of the crosslinks (i.e. polysulfide $S_{x}$, disulphide or monosulfides) can be an important parameter governing the ability of NR to crystallize.

Comparing the study of Chenal $\& \mathrm{al}^{8}$ Ikeda et al. ${ }^{7}$ and Trabelsi et al. ${ }^{3}$ seems to back up that hypothesis. The effect of changing sulphur and accelerator content does not only modify the crosslink density $v$ but also the length of the S-S crosslinking bond itself which may have an effect on crystallization

2) The chain defects should strongly affect strain-induced crystallization. This argument is based on the entropic gain brought by the strain-induced crystallization. A previous study showed that for peroxide-crosslinked rubbers, the entropy gain of strain-induced crystallization controls the crystallization threshold. ${ }^{7,15}$ The increase in sulphur induced defects along the polymer chain (dangling polysulfide chains or loops) after ageing reduces the entropy gain associated to crystallization and therefore could increase the crystallization threshold stretch.
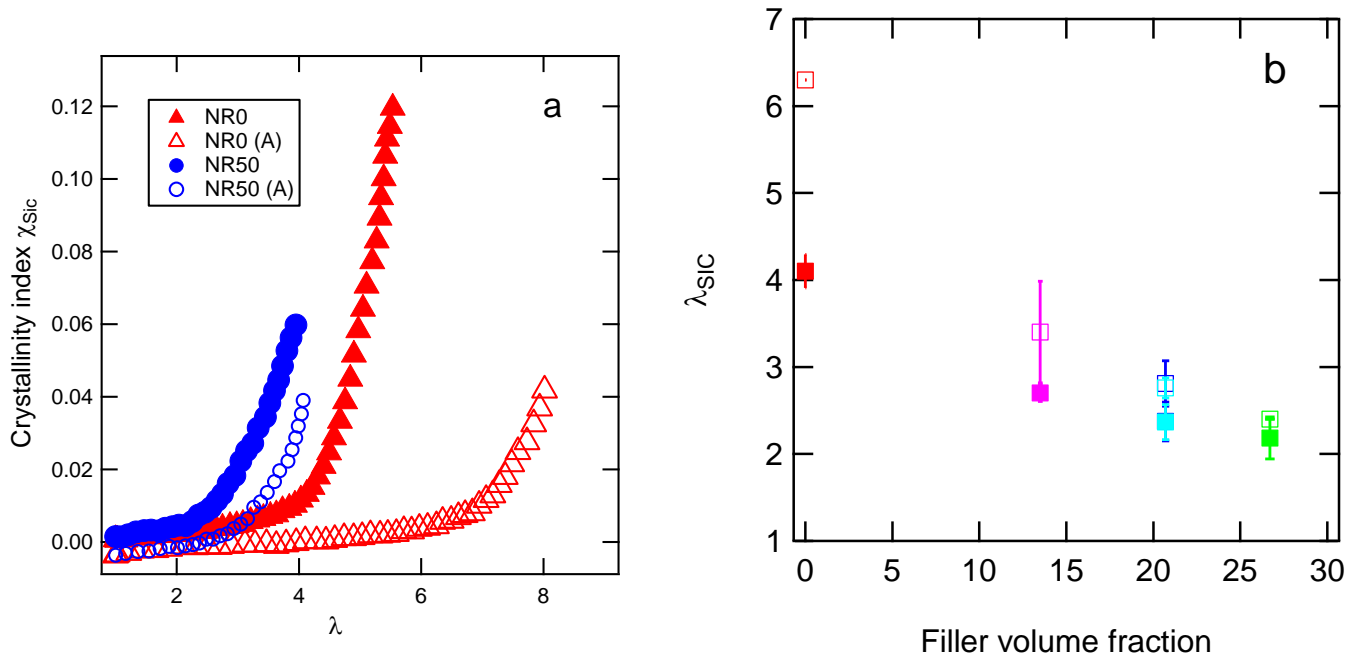

Figure $12:$ a) Crystallinity index $\chi_{\mathrm{c}}$ as a function of stretch $\lambda$ for NR0 and NR50, aged (open symbols) or pristine (closed symbols) . b) Stretch at the threshold of SIC as a function of filler volume fraction for pristine (filled symbols) and aged materials (open symbols).

The results of Figure $12 \mathrm{~b}$ can be plotted as a function of the corrected extension ratio as predicted by the Nielsen formula ${ }^{17}$ for filled materials and the results are shown on Figure 13. The corrected extension ratio at the SIC threshold is indeed higher after ageing, but no longer independent of the filler volume fraction. The effect of thermal ageing on strain-induced crystallization is clearly more pronounced when the material has a low amount of filler. This observation shows that the presence of filler reduces significantly the effects of ageing on the strain-induced crystallization, maybe through a nucleation effect that offsets the presence of chain defects. It is also possible that the presence of filler modifies the changes in Sulphur crosslinking that occur in the material during aging. 

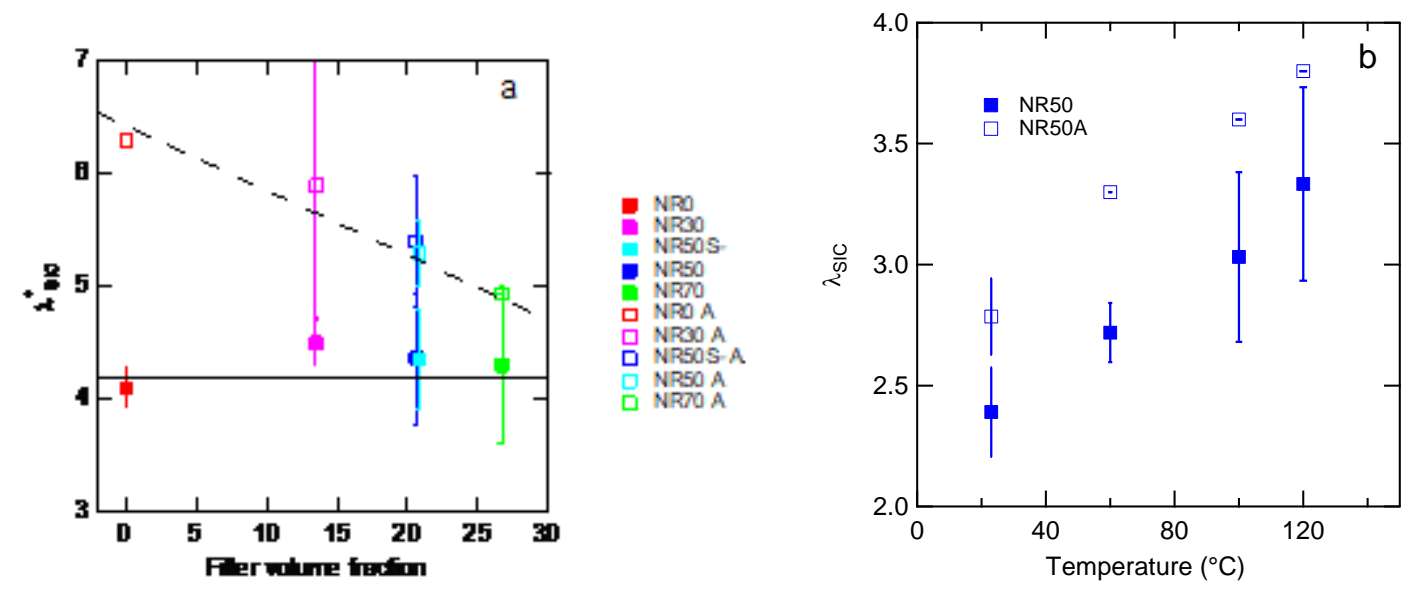

Figure $13: a$ ) Threshold value of corrected stretch for aged (open symbols) and unaged (filled symbols) materials as a function of filler volume fraction. b) SIC threshold stretches as a function of temperature for pristine NR50 (filled symbols) and NR50A, its aged counterpart (open symbols).

The delay in crystallization induced by ageing was also observed at different temperatures. As shown on Figure 13b for the reference material, temperature and ageing seems to act independently on the SIC threshold. This decreased ability to crystallize relative to the pristine material could be due to a modification of the chemical nature of the crosslink points. While conventionally cured elastomers have a majority of polysulfide links $S_{\mathrm{x}}$, the anaerobic aging process modifies progressively these polysulfide bridges into disulfide and monosulfide bridges. In addition since there is significant amount of dissolved sulfur it is likely that some loops and pendant chains can form on the polyisoprene chains and perturb the crystallinity. The constant gap observed between the new and aged threshold backs up the hypothesis that the redistribution of sulphur through ageing acts as an added energy barrier to crystallization.

\subsubsection{Ageing and cavitation}

The SAXS experiments carried out on aged materials shed also some light on the effect of thermal ageing on cavitation. The void volume fractions created under tension for both new and aged materials are displayed on Figure 14. The stress at the cavitation threshold for both aged and pristine materials seems to be nearly the same within the experimental noise. However, the void volume fraction created after the cavitation threshold is lower for aged materials at the same stress level.

This result is analogous to the result presented earlier on the effect of crosslink density on cavitation. Since uniaxial tests show that ageing reduces the average crosslink density in the materials, the change in cavitation mechanisms in aged materials could be qualitatively explained by this difference in network structure.

Table 1 shows that the average $\mathrm{M}_{\mathrm{c}}$ of the NR crosslinked with $6 \mathrm{PHR}$ of sulfur is around $9 \mathrm{~kg} / \mathrm{mole}$, while that of the NR crosslinked with 3 PHR of sulfur is around $15 \mathrm{~kg} / \mathrm{mole}$. The value of $\mathrm{M}_{\mathrm{c}}$ of the aged NR0 with 6PHR was evaluated at $11 \mathrm{~kg} /$ mole based on the decrease in modulus. Assuming that the aged NR50 has a similar reduced average crosslink density, it is logical to find that its cavitation behavior lies between that of NR50 and that of NR50 S-.

This increase in volume fraction of cavities with increasing crosslink density can be rationalized in the following way. As first proposed by Gent and Wang ${ }^{41}$ and analysed in more detail by Lin and $\mathrm{Hui}^{42}$, the formation of cavities in a crosslinked rubber is an irreversible fracture process. The internal pressure required for the growth of a cavity by fracture depends on the ratio between the Fracture Energy $\Gamma$ of the material and its Young's modulus $E .^{39}$. In the case of filled elastomers in uniaxial tension, the cavities open because of the presence of confined regions between, filler particles or 
clusters. These cavities do not grow in size because the confined regions (where there is a driving force for cavitation) are small. It was shown by Zhang that the biggest cavities open first ${ }^{22,35}$. The difference between the volume fractions of nucleated cavities observed in Figure 14 suggests that the smaller cavities are not opened in the less crosslinked material, which must have therefore a higher value of $\Gamma / E$. This is consistent with the increase in fracture energy and decrease in modulus predicted by the Lake-Thomas theory ${ }^{43}$ and observed experimentally ${ }^{44}$.

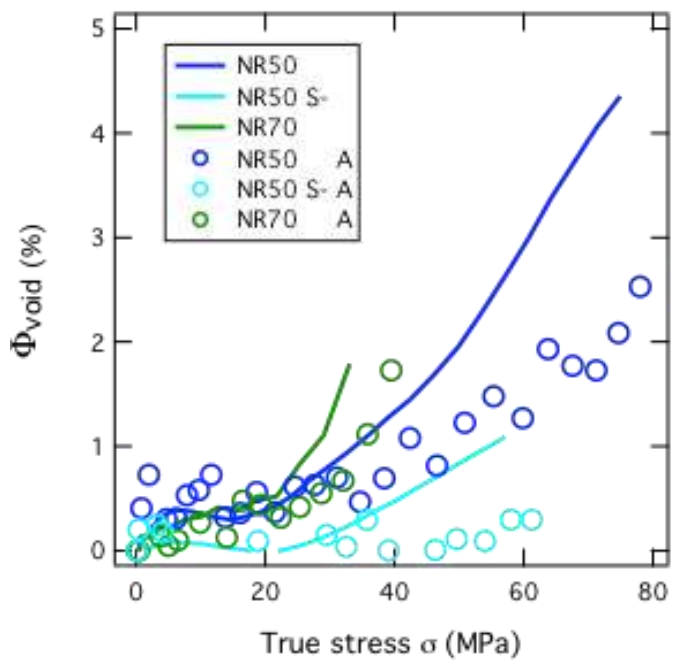

Figure 14 : Volume fraction of voids observed during the first loading in filled natural rubber for pristine and aged materials $(A)$

\section{Concluding remarks}

We have extensively investigated and for the first time, the modifications in structure of highly crosslinked and filled natural rubber occurring during in situ stretching and in particular at temperatures above room temperature and up to $120^{\circ} \mathrm{C}$. Since this type of material is typically used in truck tyres we also investigated the change in structure and its consequences on the mechanical behavior for materials aged for 10 days in anaerobic conditions. The occurrence of strain-induced crystallization was investigated by WAXS while the appearance of nanocavities at high stretch levels was detected by SAXS by monitoring changes in the scattering invariant.

Several conclusions can be drawn from the work. First of all the presence of the carbon black filler, as reported in previous studies, dramatically improves the mechanical properties at high temperature and reduces the threshold stretch $\lambda_{\text {SIC }}$ for the occurrence of strain induced crystallization. This increase in $\lambda_{\text {sIC }}$ can be qualitatively and also quantitatively understood if the nominal stretch of the filled elastomer is renormalized with Nielsen's equation effectively applying an amplification factor. Still most remarkably we find that the filled natural rubber with 21 vol\% of CB can still crystallize under strain at $120^{\circ} \mathrm{C}$ before breaking. We also find, as previously reported, that the presence of filler triggers the nucleation of nanocavities at high stretch which appear only above a true stress of $25 \mathrm{MPa}$ for all materials. However the threshold stress of cavitation increases significantly with temperature, and reaches a true stress of $70 \mathrm{MPa}$ at $100^{\circ} \mathrm{C}$. Since the cavitation threshold of an unfilled rubber decreases with increasing $\mathrm{T}$, this new result strongly supports the argument that cavitation occurs in the confined material between fillers. An increase in temperature decreases the thickness of the immobilized polymer layer around the filler and reduces the local confinement. 
Finally aging of the materials in anaerobic conditions modifies the threshold for the onset of SIC and for cavitation. Both processes occur for higher extensions and this effect is attributed to modifications of the network structure leading to a change in the localization of sulfur in the rubber, with Polysulfidic crosslinks being modified into a lower density of mono or disulfidic crosslinks and the excess sulfur forming dangling short chains or loops on the main elastomeric chain, The reduction of the modulus of the elastomer increases the cavitation threshold while the side chains of sulfur may be responsible for the increase in threshold for SIC.

\section{Acknowledgements}

We are grateful to all the support teams of the synchrotron beam lines. In particular we thank Cyrille Rochas at BM02 at the European Radiation Synchrotron Facility (Grenoble, France), Steven Weigand and Josh Yeh at the 5ID-D line of the Advanced Photon Source (Argonne, USA) (two runs), and Javier Perez at the SWING beamline of the SOLEIL synchrotron source (Saclay, France). Yann Auriac from Centre des Matériaux is acknowledged for his help in designing the furnace for the in-situ tensile stage for ESRF and SOLEIL.

\section{References}

Nie, Y.; Gu, Z.; Wei, Y.; Hao, T.; Zhou, Z. Polym J 2017, 49, 309-317.

Huneau, B. Rubber Chemistry and Technology 2011, 84, 425-452.

Trabelsi, S.; Albouy, P. A.; Rault, J. Macromolecules 2003, 36, 7624-7639.

Candau, N.; Laghmach, R.; Chazeau, L.; Chenal, J. M.; Gauthier, C.; Biben, T.; Munch, E. Macromolecules 2014, 47, 5815-5824.

Toki, S.; Sics, I.; Ran, S. F.; Liu, L. Z.; Hsiao, B. S.; Murakami, S.; Senoo, K.; Kohjiya, S. Macromolecules 2002, 35, 6578-6584.

Toki, S.; Hsiao, B. S.; Amnuaypornsri, S.; Sakdapipanich, J. Polymer 2009, 50, 2142-2148.

Ikeda, Y.; Yasuda, Y.; Hijikata, K.; Tosaka, M.; Kohjiya, S. Macromolecules 2008, 41, 5876-5884.

Chenal, J. M.; Gauthier, C.; Chazeau, L.; Guy, L.; Bomal, Y. Polymer 2007, 48, 6893-6901.

Candau, N.; Chazeau, L.; Chenal, J.-M.; Gauthier, C.; Munch, E. Composites Science and Technology 2015, 108, 9-15.

Rault, J.; Marchal, J.; Judeinstein, P.; Albouy, P. A. Macromolecules 2006, 39, 8356-8368.

Spratte, T.; Plagge, J.; Wunde, M.; Klüppel, M. Polymer 2017, 115, 12-20.

Bruning, K.; Schneider, K.; Roth, S. V.; Heinrich, G. Macromolecules 2012, 45, 7914-7919.

Beurrot-Borgarino, S.; Huneau, B.; Verron, E.; Rublon, P. International Journal of Fatigue 2013, 47, 17.

Candau, N.; Laghmach, R.; Chazeau, L.; Chenal, J.-M.; Gauthier, C.; Biben, T.; Munch, E. European Polymer Journal 2015, 64, 244-252.

Ikeda, Y.; Yasuda, Y.; Makino, S.; Yamamoto, S.; Tosaka, M.; Senoo, K.; Kohjiya, S. Polymer 2007, 48, 1171-1175.

Tosaka, M.; Kohjiya, S.; Murakami, S.; Poompradub, S.; Ikeda, Y.; Toki, S.; Sics, I.; Hsiao, B. S.

Rubber Chemistry and Technology 2004, 77, 711-723.

Nielsen, L. E. Journal of Applied Polymer Science 1966, 10, 97-103.

Zhang, H.; Scholz, A. K.; Merckel, Y.; Brieu, M.; Berghezan, D.; Kramer, E. J.; Creton, C. Journal of Polymer Science Part B: Polymer Physics 2013, 51, 1125-1138.

Candau, N.; Laghmach, R.; Chazeau, L.; Chenal, J.-M.; Gauthier, C.; Biben, T.; Munch, E. Polymer 2015, 60, 115-124. 
Toki, S.; Che, J.; Rong, L.; Hsiao, B. S.; Amnuaypornsri, S.; Nimpaiboon, A.; Sakdapipanich, J. Macromolecules 2013, 46, 5238-5248.

de Crevoisier, J.; Besnard, G.; Merckel, Y.; Zhang, H.; Vion-Loisel, F.; Caillard, J.; Berghezan, D.; Creton, C.; Diani, J.; Brieu, M.; Hild, F.; Roux, S. Polymer testing 2012, 31, 663-670.

Zhang, H.; Scholz, A. K.; Vion-Loisel, F.; Merckel, Y.; Brieu, M.; Brown, H.; Roux, S. p.; Kramer, E. J.; Creton, C. Macromolecules 2013, 46, 901-913.

Zhang, H.; Scholz, A. K.; de Crevoisier, J.; Berghezan, D.; Narayanan, T.; Kramer, E. J.; Creton, C. Journal of Polymer Science Part B: Polymer Physics 2015, 53, 422-429.

Le Gac, P. Y.; Broudin, M.; Roux, G.; Verdu, J.; Davies, P.; Fayolle, B. Polymer 2014, 55, 2535-2542. Rublon, P., 2013. Ecole Centrale de Nantes (ECN) (ECN) (ECN) (ECN), 2013.

Pannier, Y.; Proudhon, H.; Mocuta, C.; Thiaudiere, D.; Cantournet, S. Journal of Synchrotron Radiation 2011, 18, 907-911.

Poompradub, S.; Tosaka, M.; Kohjiya, S.; Ikeda, Y.; Toki, S.; Sics, I.; Hsiao, B. S. Journal of Applied Physics 2005, 97, 103529.

Murakami, S.; Senoo, K.; Toki, S.; Kohjiya, S. Polymer 2002, 43, 2117-2120.

Trabelsi, S.; Albouy, P.-A.; Rault, J. Macromolecules 2002, 35, 10054-10061.

Dumbleton, J. H.; Bowles, B. B. Journal of Polymer Science Part A-2: Polymer Physics 1966, 4, 951958.

Albouy, P. A.; Marchal, J.; Rault, J. European Physical Journal E 2005, 17, 247-259.

Saintier, N.; Cailletaud, G.; Piques, R. Materials Science and Engineering: A 2011, 528, 1078-1086.

Rublon, P.; Huneau, B.; Verron, E.; Saintier, N.; Beurrot, S.; Leygue, A.; Mocuta, C.; Thiaudière, D.;

Berghezan, D. Engineering Fracture Mechanics 2014, 123, 59-69.

Hernández, M.; López-Manchado, M. A.; Sanz, A.; Nogales, A.; Ezquerra, T. A. Macromolecules 2011, 44, 6574-6580.

Zhang, H.; Scholz, A. K.; de Crevoisier, J.; Vion-Loisel, F.; Besnard, G.; Hexemer, A.; Brown, H. R.; Kramer, E. J.; Creton, C. Macromolecules 2012, 45, 1529-1543.

Bueche, F. Journal of Applied Polymer Science 1960, 4, 107-114.

Rublon, P.; Huneau, B.; Saintier, N.; Beurrot, S.; Leygue, A.; Verron, E.; Mocuta, C.; Thiaudière, D.; Berghezan, D. Journal of Synchrotron Radiation 2013, 20, 105-109.

Hernández, M.; Sanz, A.; Nogales, A.; Ezquerra, T. A.; López-Manchado, M. A. Macromolecules 2013, 46, 3176-3182.

Cristiano, A.; Marcellan, A.; Long, R.; Hui, C. Y.; Stolk, J.; Creton, C. Journal of Polymer Science Part B: Polymer Physics 2010, 48, 1409-1422.

Montes, H.; Lequeux, F.; Berriot, J. Macromolecules 2003, 36, 8107-8118.

Gent, A. N.; Wang, C. Journal of Materials Science 1991, 26, 3392-3395.

Lin, Y. Y.; Hui, C. Y. International Journal of Fracture 2004, 126, 205-221.

Lake, G. J.; Thomas, A. G. Proceedings of the Royal Society of London, series A: Mathematical and Physical Sciences 1967, A300, 108-119.

Mzabi, S.; Berghezan, D.; Roux, S.; Hild, F.; Creton, C. Journal of Polymer Science: Polymer Physics $2011,49,1518-1524$. 